\title{
Velocity measurements in regions of upstream influence of a body in aligned-fields MHD flow
}

\author{
By BRUCE M. LAKE† \\ California Institute of Technology, Pasadena, California
}

(Received 17 March 1971 and in revised form 1 June 1971)

Experiments are described in which velocities were measured ahead of a semiinfinite Rankine body moving parallel to a uniform magnetic field in a conducting fluid. The flow disturbance in front of the body is found to increase in length as $N^{\frac{1}{2}}$, where $N$ is the interaction parameter. In most of the experiments this parameter was varied from 4 to about 50. Measurements made along the axis of symmetry in the flow show that there is a relatively short region of stagnant fluid directly ahead of the body. The major part of the disturbance is found to consist of a much longer region in which the flow undergoes transition from conditions in the free stream to conditions near the body. Velocity profiles across the flow in this region show that for increased $N$, at a fixed distance ahead of the body, the velocity defect increases and the disturbance becomes more confined radially. Although the radial gradients in the flow increase with $N$, they are found to be much smaller than would be expected in a flow containing thin current layers. A physical model of the flow which has currents and pressures consistent with these results is discussed.

\section{Introduction}

Although aligned-fields MHD flow past bodies has been the subject of considerable theoretical work, the solutions which have been found correspond to cases which cannot be realized in the laboratory. This can be seen by comparing the conditions assumed in the various theories with those appropriate to laboratory flows, as has been done by Yonas (1966). In general, the results of such a comparison show that theoretical studies of MHD flow over bodies treat the fluid as either highly viscous or highly conducting, whereas the liquid metals in which the experiments must be performed are fluids of relatively small kinematic viscosity and electrical conductivity.

The equations of motion for the limits appropriate to the experimental flow are simple in appearance, but the analytic solution of them remains a difficult non-linear problem. The dimensionless equations governing the steady laminar flow of a viscous incompressible conducting fluid are (Shereliff 1965)

$$
\begin{gathered}
\nabla \cdot \mathbf{q}=0, \\
(\mathbf{q} \cdot \nabla) \mathbf{q}=-\nabla p+N(\mathbf{j} \times \mathbf{B})+(\mathbf{l} / R e) \nabla^{2} \mathbf{q},
\end{gathered}
$$

$\dagger$ Present address: TRW Systems, Redondo Beach, California. 


$$
(\mathbf{q} . \nabla) \mathbf{B}-(\mathbf{B} \cdot \nabla) \mathbf{q}=(\mathbf{1} / R m) \nabla^{2} \mathbf{B}
$$

where $\quad \mathbf{x}=\frac{\mathbf{x}^{*}}{d}, \quad \mathbf{q}=\frac{\mathbf{q}^{*}}{U}, \quad \mathbf{B}=\frac{\mathbf{B}^{*}}{B_{0}}, \quad \mathbf{j}=\frac{\mathbf{j}^{*}}{\sigma U B_{0}}, \quad p=\frac{p^{*}-p_{1}}{\rho U^{2}}$,

and $R e=U d / \nu$, the Reynolds number; $N=\sigma B_{0}^{2} / \rho U$, the interaction parameter; and $R m=\mu \sigma U d$, the magnetic Reynolds number. In the experiments described here, and in other experiments to which reference will later be made, laboratory conditions correspond to

$$
10^{3}<R e<10^{5}, \quad 0 \leqslant N<10^{2}, \quad R m\left\{\begin{array}{l}
\ll 1 \text { in } \mathrm{Hg}, \\
\leqslant O(1) \text { in Na. }
\end{array}\right.
$$

Therefore, in terms of these parameters, the experimental limits are $R m \leqslant O(1)$, $R e \gg 1$, and $N \ll R e$, whereas the theoretical limits referred to above correspond to $R m \gg 1$ and $N \gg R e$.

Two assumptions can be made which simplify the equations governing the experimental flow. The first, that the flow is inviscid, follows from the fact that $R e \gg 1$, and the drag measurements of Suzuki (1967), which indicate that the flow over this Rankine body under these conditions is laminar and unseparated. The second, that $\mathbf{B}^{*}=-B_{0} \mathbf{i}$, follows from $R m \ll 1$, using Ampere's law, $\nabla \times \mathbf{B}=R m \mathbf{j}$, and Ohm's law, $\mathbf{j}=\mathbf{q} \times \mathbf{B}, \dagger$ which imply that $\Delta \mathbf{B}=O(R m)$ when $\mathbf{B}, \mathbf{q}=O(1)$. The induced magnetic fields can, therefore, be neglected, uncoupling the momentum and induction equations and making the current density simply $\mathbf{j}=-\mathbf{q} \times \mathbf{i}=v \mathbf{i}_{\theta}$. The equations of motion, in cylindrical coordinates become

$$
\begin{aligned}
& \frac{\partial u}{\partial x}+\frac{\partial v}{\partial r}+\frac{v}{r}=0, \\
& u \frac{\partial u}{\partial x}+v \frac{\partial u}{\partial r}=-\frac{\partial p}{\partial x}, \\
& u \frac{\partial v}{\partial x}+v \frac{\partial v}{\partial r}=-\frac{\partial p}{\partial r}-N v,
\end{aligned}
$$

where, for these experiments, $N \geqslant O(1)$. For $N \gg 1$, the inertia terms in the radial momentum equation may be neglected. The radial pressure gradient is then maintained by the magnetic force. The current flows in rings, $\mathbf{j}=v \mathbf{i}_{\theta}$, and the magnetic force both opposes the radial flow and is proportional to it.

A model for this flow in which the currents ahead of the body exist in relatively thin layers concentrated about $r=r_{\text {body }}=\frac{1}{2}$ was suggested by Childress $(1963 b)$. Yonas (1966) and Suzuki (1967) scale the variables in these equations (for $N \gg 1$ ) to show that in such layers, for $p, u=O(1)$ and $O(1) \leqslant x \leqslant O(N)$, the equations can be satisfied for $\tilde{\gamma}=r-\frac{1}{2}=O(x / N)^{\frac{1}{2}} \S$ and $v=j=O\left(1 /(N x)^{\frac{1}{2}}\right)$. These layers would, therefore, extend upstream from the body, growing like $N^{\frac{1}{2}}$, separating

$\dagger E=0$ in axisymmetric flows.

$\ddagger$ Although, as stated, this approximation would seem to be reasonable only for mercury flows, it should apply to flows in sodium as well (where $R m \leqslant O(1)$ ), because Childress $(1963 b)$ has shown that, for $N \gg 1, \Delta \mathbf{B}=O(R m / N)$.

$\S$ Defined in this way, $\tilde{r}$ is the radial co-ordinate for thin current layers which are centred about $r=\frac{1}{2}$. 
stagnated flow ahead of the body from the free-stream flow outside the layers, and merging at $x=O(N)$. Such layer solutions, when found, would then have to be matched to solutions in adjacent flow regions, including an intermediate upstream region joining the layers at $x=O(N)$ and the flow at upstream infinity. No such solutions have been found, however, since the same non-linear equations (with $\partial \tilde{p} / \partial \tilde{r}=-\tilde{v}$ in scaled variables) apply in the various regions. The current layer model is mentioned here because it makes qualitative predictions about the flow which can be tested by experiment.

In 1960, when the GALCIT mercury tow tank facility was constructed, there were no known experimental investigations of these flows. Several experiments have since been performed. Maxworthy (1962) has measured the drag of freely falling spheres in liquid sodium, and more recently $(1968,1969)$ the pressure distribution around a sphere and sphere with finite afterbody in a liquid sodium tunnel. Yonas (1966) has measured the drag of spheres and of a flat disk in the same liquid sodium tunnel. In the GALCIT facility, Ahlstrom (1963) has measured the magnetic field perturbations produced ahead of a semi-infinite Rankine body, and Suzuki (1967) has measured the drag on a semi-infinite Rankine body. The results of the above experiments which apply to the flow conditions of the measurements presented in this paper are discussed briefly here and in more detail in Lake (1969).

Previous experimental investigations of aligned-fields MHD flow past bodies have not provided direct measurements of the actual fluid motions in such flows. The experiments described here were undertaken to provide a description of the fluid motions by means of velocity measurements. These were made using electrically insulated hot-film sensors in the flow ahead of a semi-infinite Rankine body.

\section{Experiment}

\subsection{Apparatus}

These experiments were performed in the GALCIT mercury tow tank facility, which is basically a mercury-filled cylindrical stainless steel tank surrounded by a water-cooled copper solenoid capable of producing steady axial magnetic fields of up to 12 kilogauss in strength. The tank is mounted with its axis vertical and is $55 \mathrm{in}$. long and $5.5 \mathrm{in}$. in internal diameter. The magnetic fields were calibrated using a Hall-effect probe and found to be uniform axially to $5 \%$ and uniform radially to $2 \%$ in the middle 25 in. of the solenoid.

Models are driven through the mercury along the axis of the tank on the end of a 1 in. outer diameter stainless steel shaft which passes through graphitar bearings at the tank bottom. A system of cables with a magnetic cluteh and brake connect the lower end of this drive shaft to a variable speed transmission and an electric motor. The shaft accelerates to a constant velocity within 2 to 3 in. and can be driven at speeds of up to $3 \mathrm{~m} / \mathrm{sec}$. The total length of travel was usually from 29 to $36 \mathrm{in}$. The drive shaft velocity is measured by a velocity servo which is simply a rotating potentiometer geared to the cable drive so as to produce a voltage output which, when electronically differentiated, is directly 
proportional to shaft velocity. This facility has been described in detail by Liepmann, Hoult \& Ahlstrom (1962). A schematic diagram of the overall facility as used in these experiments can be found in Lake (1969).

The velocity measurements were made using a hot-film sensor manufactured by Thermo-Systems Inc. of Minneapolis. It consists of a platinum film of approximately $10^{3} \AA$ thickness deposited onto a $0.002 \mathrm{in}$. diameter quartz cylinder and insulated with a sputtered quartz coating approximately $1.6 \times 10^{4} \AA$ thick. The cylinder has an overall length of $0.080 \mathrm{in}$. and a sensitive length of $0.040 \mathrm{in}$. The length-to-diameter ratio is therefore 20 to 1 . It is supported by epoxy-coated needles which are mounted on the end of a 0.125 in. diameter stainless steel shaft. The opposite end of the shaft has an O-ring seal and gold-plated electrical contact pins so that the probe may be attached to various holders with a mercury-tight fit. The temperature coefficient of resistance of each sensor was found experimentally, a typical value being $0.00256^{\circ} \mathrm{C}^{-1}$. The successful application of this type of hot-film sensor to the measurement of turbulence intensities in mercury has been reported by Malcolm (1969).

The model used for these experiments was a lucite Rankine half-body mounted on the end of the tow tank drive shaft to simulate a semi-infinite body. This body shape, which is defined by a source in a uniform stream, was chosen because the magnetic field induced by it had been measured previously by Ahlstrom (1963), and the drag force on it had been measured by Suzuki (1967). Their choice of the Rankine half-body was motivated primarily by the simple analytical form of its potential flow.

\subsection{Procedure}

Measurements were made with the sensor at the lower end of a $0 \cdot 180 \mathrm{in}$. diameter stainless steel probe, which was suspended above the free surface of the mercury at the top of the tow tank (figure 1 $(a)$ ). The holder to which the upper half of the probe's 24 in. length was attached, allowed the probe to be moved vertically or horizontally for positioning of the sensor in the mercury, and held it firmly in place when positioned. The range of horizontal sensor movement was limited to $r \leqslant 1.25$ in. When mounted in this way, the sensor measured the flow velocity induced by the approaching model at a fixed point in the laboratory reference frame.

Measurements were also made with the sensor at the end of another 0.180 in. diameter stainless steel probe which was attached to the end of the tow tank drive shaft (figure $1(b)$ ). In this arrangement the electrical connexions were made by means of a cable brought up through the hollow drive shaft. A Rankine body headform was then fitted to the end of the sting so that, again, the sting and model formed a semi-infinite body, this time with the sensor and probe mounted on the body. The distance between the sensor and body stagnation point was adjusted by means of cylindrical sections of various lengths which were put between the end of the drive shaft and the model headform. In this way velocity measurements were made with the sensor at a fixed position ahead of the body during the entire course of a run.

The sensor was operated at constant temperature using a Thermo-Systems Model $1000 \mathrm{~A}$ anemometer. The anemometer signal was fed through a voltage- 
to-frequency converter to a digital counter so that fluctuations as small as one part in 10000 could be monitored. During the course of a run, the outputs from the anemometer and the drive shaft velocity servo were displayed simultaneously on a dual-beam oscilloscope and their traces recorded photographically.

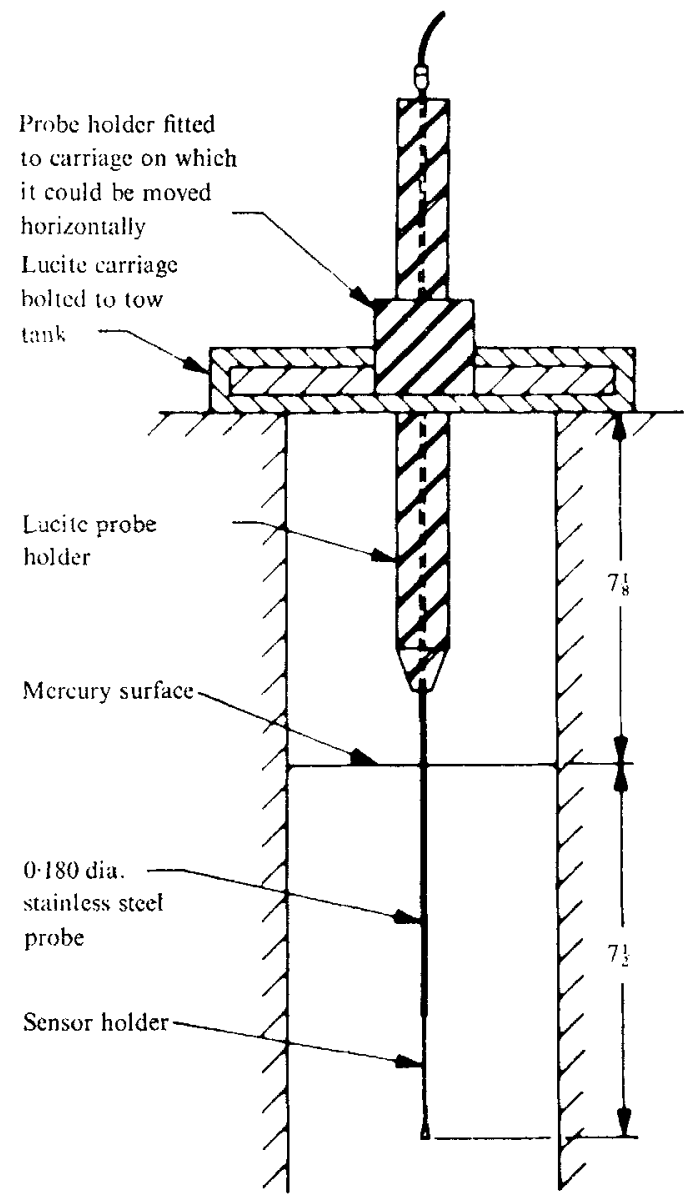

(ii)

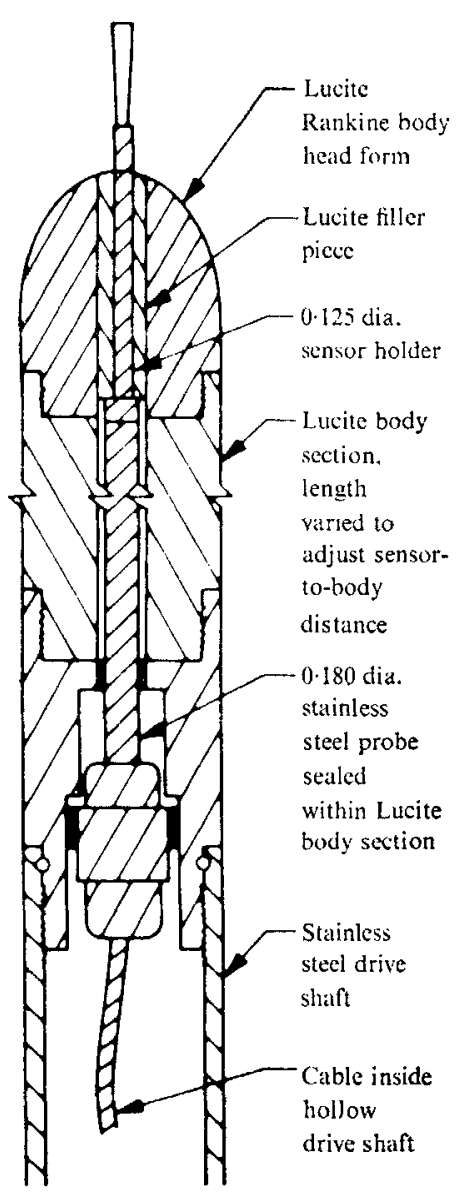

(b)

FIGURE 1. Sensor mounting positions in the tow tank. (a) Tank-mounted sensor, $(b)$ shaft-mounted sensor.

The actual operating procedure used during the course of the experiments was determined primarily by the unique difficulties encountered when hot films of this kind are used in liquid mercury. It is impossible to keep a free surface of mercury perfectly clean, and when a sensor is passed through such a surface the quartz insulation, which is not wetted by the mercury, picks up a coating of impurities. The hot film is then effectively surrounded by both a layer of quartz and another layer of unknown composition. As long as this impurity coating remains unchanged, its effect on the performance of the sensor is not bothersome. This is the case during the course of a given immersion, except that even at zero velocity and constant temperature the anemometer signal may exhibit a slow 
drift. The possible occurrence of this kind of drift, which Sajben (1965) attributes to the presence of minute amounts of impurities within the mercury, was one reason the anemometer signal was accurately monitored at all times.

More important is the fact that the properties of the impurity coating may change significantly each time the sensor is passed through the mercury surface. As a result, a sensor operated under otherwise identical conditions during different immersions, may perform as though the sensor-to-fluid temperature difference, or overheat, had been altered. Although this effect of the impurity coating can be eliminated by calibrating in terms of the difference between heat transfer at any velocity and that at some reference velocity (zero velocity was used), such a calibration will be in error unless both measurements used to calculate the difference are made under conditions for which only the velocity has changed. In particular, quantities such as the properties of the sensor impurity coating and the fluid temperature, as well as the various directly controllable quantities involved, must be identical. To ensure that this was the case, the mercury temperature and the anemometer signal for zero velocity were measured before and after every run. In addition, a vacuum skimmer was used to clean the mercury surface before removing or inserting the sensor, and the number of times the sensor was passed through the surface was kept at a minimum. Finally, whenever possible, the sensor was recalibrated after each new immersion.

When measurements were made with the sensor tank-mounted, it was calibrated using the rising displacement flow produced in the mercury by the drive shaft as it entered from below. The velocity profile of this flow was measured and found to be quite flat at the level of the sensor. The velocity of the displacement flow was calculated using the measured drive shaft velocity and the cross-sectional areas of the tank and the drive shaft. In this way the sensor was calibrated over a velocity range of from $0 \cdot 3$ to $4 \mathrm{~cm} / \mathrm{sec}$. When data runs were made with the sensor mounted in this way and positioned within one body radius of the tow tank centre-line, the drive shaft stops were set so that the stagnation point of the Rankine body was $0.5 \mathrm{in}$. from the sensor at the end of each run. For this reason, measurements of the potential flow of the Rankine body at zero magnetic field were of little value, for while they reproduced the known velocity profile to within $0.5 \mathrm{in}$. of the stagnation point, more than $85 \%$ of the overall velocity change in the potential flow velocity field occurs in the final $0.5 \mathrm{in}$. At low speeds it was possible to allow the shaft to run directly into these solid stops, but at higher speeds a passive drive motor disconnect switch was required to allow the shaft to decelerate before impacting the solid stops. When used, this switch was located $2 \mathrm{in}$. ahead of the solid stops so that useful data were obtained only to within $2.5 \mathrm{in}$. of the model. During runs for which the sensor was positioned more than one body radius off the tank centre-line, the body was allowed to travel past the sensor.

When the magnetic field was turned on, the temperature of the entire system rose due to the heat generated in the solenoid. This temperature change was monitored using thermocouples at various locations in the solenoid cooling system, and the sensor itself in the mercury. It was found that waiting periods of well over an hour were required to make sure that the temperature of the system 
had completely stabilized. After taking data over the full range of driveshaft velocities, the magnetic field was turned off and the system was allowed to return to room temperature. The sensor was then recalibrated before it was removed from the mercury. This procedure was followed for each value of the magnetic field.

When measurements were made with the single sensor offset radially from the flow centre-line, runs were made with it oriented both parallel and perpendicular to the tank radius as a check on the possible effects of whatever small radial velocity was present.

When the sensor was mounted on the drive shaft, it was positioned from 4 to $10 \mathrm{in}$. ahead of the body for all calibration runs. The velocity at the sensor was assumed to be that of the drive shaft minus that of the displacement flow. This assumption is supported by zero field measurements which show no change in displacement flow velocity as the model approached to within $4 \mathrm{in}$. of the tankmounted sensor and by the fact that the potential flow disturbance is less than $0.4 \%$ of the body speed at a distance of 4 in. The calibration velocity range was from $2.5 \mathrm{~cm} / \mathrm{sec}$ to more than $20 \mathrm{~cm} / \mathrm{sec}$.

Data were taken at a fixed value of the magnetic field for the full range of velocities at each of five sensor positions: 4 in., 3 in., $2 \mathrm{in} ., 1 \mathrm{in}$. and $0.5 \mathrm{in}$. ahead of the body stagnation point. This involved removing the sensor each time its position was changed. Data were also taken with the sensor at one fixed position ahead of the body for a range of velocities at each of several values of the magnetic field. This produced measurements at one position over the full range of flow conditions without passing the sensor through the mercury surface, but was extremely inefficient due to the number of time-consuming magnetic field changes required. Only enough runs were made using this latter procedure to confirm that the difference between the data from the two procedures was not greater than the uncertainty in the calibration itself. This indicates that the frequent immersions did not tend to produce a more unstable impurity layer which could change significantly during the course of a run.

For these experiments the range of body velocities was from about $2 \mathrm{~cm} / \mathrm{sec}$ to over $20 \mathrm{~cm} / \mathrm{sec}$. The magnetic fields used were $4500,6800,9000$ and 11300 gauss. The ranges of the basic dimensionless parameters were therefore as follows:

$$
5 \times 10^{3} \leqslant R e \leqslant 9 \times 10^{4}, \quad 7 \times 10^{-4} \leqslant R m \leqslant 1 \times 10^{-2}, \quad 4 \leqslant N \leqslant 84,
$$

and for all the runs the Alfvén number, $\alpha=B_{0} / U(\rho \mu)^{\frac{1}{2}}$, was greater than unity.

\subsection{Data reduction}

The sensors were calibrated using a technique developed by Sajben (1965) to account for the effects of the quartz coating and the impurity layer on the measured heat-transfer rate $q$. The expression for the rate of heat transfer for the case of a very long uniformly heated composite cylinder in a steady uniform flow perpendicular to its axis can be written $\dagger$

$$
\frac{\pi k_{f} L\left(T_{\text {film }}-T_{\text {fluid }}\right)}{q}=\frac{1}{N u}+\frac{1}{2} \frac{k_{f}}{k_{c}} \ln \left(r_{c} / r_{\text {film }}\right)+\frac{1}{2} \frac{k_{f}}{k_{i}} \ln \left(r_{i} / r_{c}\right)
$$

$\dagger$ Where $k$ is thermal conductivity, $L$ is cylinder length, $T$ is temperature, $r$ denotes radial distance from the cylinder axis, and $N u$ is Nusselt number based on $r$. The subscripts $f, c$, and $i$ refer to the fluid, the quartz coating, and the impurity coating, respectively. 
From this equation it can be seen that even for a fixed film-to-fluid temperature difference and constant fluid properties, changes in the measured quantities on the left-hand side correspond to changes in the velocity dependent term, $1 / N u(P e), \dagger$ only if the two right-hand terms, which depend on the properties of the insulating layers, are constant. Sajben (1965) has shown that these velocity independent terms can be eliminated by defining

$$
X(P e)=\left[\pi k_{f} L\left(T_{\text {film }}-T_{\text {fluid }}\right)\right]\left[\frac{1}{q(0)}-\frac{1}{q(P e)}\right]=\frac{1}{N u(0)}-\frac{1}{N u(P e)} .
$$

A calibration in terms of $X(P e) v s . P e$ does not depend on the properties of the insulating layers as long as each $X(P e)$ value is calculated using a pair of measurements at $P e=0$ and $P e \neq 0$ for which the properties of the layers are identical. In addition, each pair of measurements must be made at the same film-to-fluid temperature difference and at the same fluid temperature.

$X(P e)$ was obtained using the ohmic dissipation calculated from the measured values of sensor voltage and resistance. Because a computer was used to calculate $X(P e), P e$ and all other flow parameters, it was possible to evaluate them using the values of the fluid properties which corresponded to the measured temperature of each run. Calibration curves were used to find $P e$ using the values of $X(P e)$ determined from the quantities measured during the data runs. $X(P e)$ vs. $P e$ calibrations for the two velocity ranges covered in these experiments can be found in Lake (1969).

The velocity measured by the sensor, the velocity of the displacement flow, and the velocity of the drive shaft were used to calculate a normalized velocity $u$ such that $u=0$ in the undisturbed displacement flow and $u=1$ at the body stagnation point. In a tow tank without a displacement flow, $u$ would be the ratio of flow velocity to body velocity measured by an observer fixed in the laboratory co-ordinate system. It is the normalized form of the velocity perturbation produced by the moving body. For measurements made with the sensor mounted at the top of the tow tank,

$$
u=\frac{P e_{\text {measured }}-P e_{\text {displ. Plow }}}{P e_{\text {drive shatt }}-P e_{\text {displ. plow }}} .
$$

For measurements made with the sensor mounted on the drive shaft,

$$
u=\frac{P e_{\text {drive shaft }}-P e_{\text {measured }}-P e_{\text {dispi. llow }}}{P e_{\text {drive shaft }}-P e_{\text {displ. flow }}} .
$$

Each tank-mounted sensor run produced data from which a full profile of flow velocity versus axial distance could be obtained. Each shaft-mounted sensor run supplied data leading to only one point in such a profile.

\section{Results}

\subsection{Velocity measurements}

Results of typical measurements of axial velocity on the flow centre-line ahead of the Rankine body which were made with the sensor mounted at the top of the tow tank are shown in figure 2. The normalized velocity $u$ is plotted as a 
function of distance in body diameters from the body stagnation point for various values of the interaction parameter. The zero magnetic field Rankine body axial velocity profile is included for comparison. The measurements made with the sensor mounted on the drive shaft did not provide complete profiles of this kind

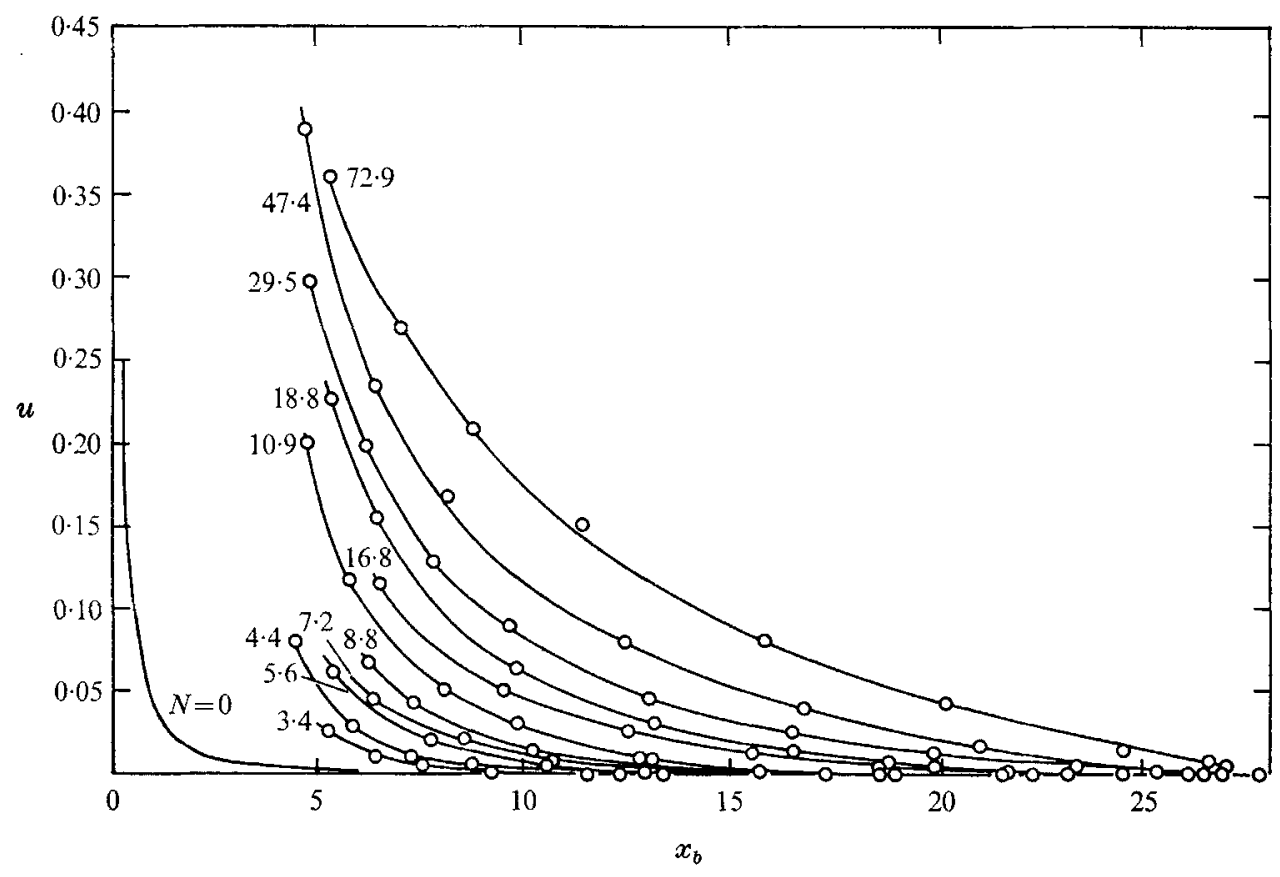

FIGURE 2. Normalized velocity on the flow centre-line vs. distance from body for a range of interaction parameters.

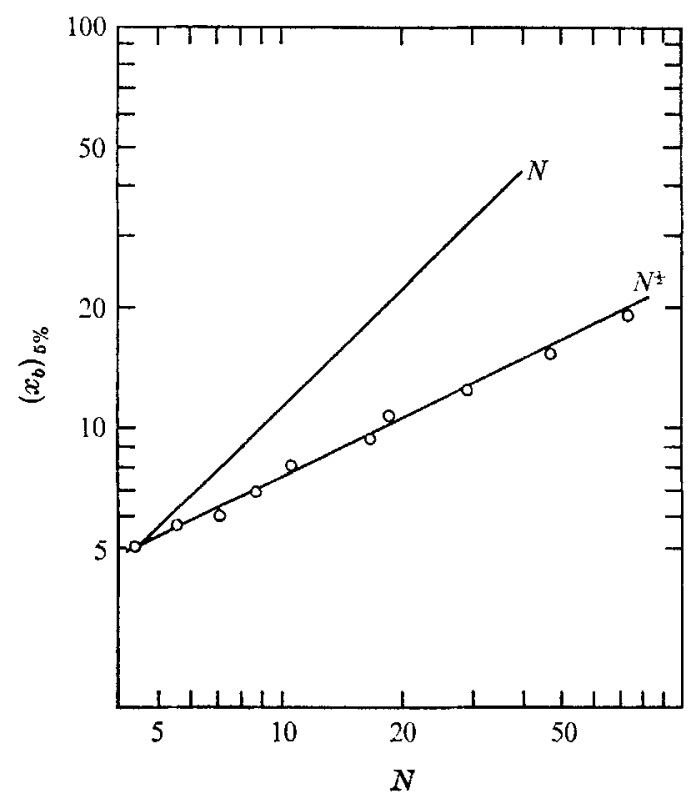

Frguke 3. Disturbance length vs. interaction parameter. 
because each run produced a data point at a slightly different value of the interaction parameter, and the range of distances from the body was not as great.

The axial length of the upstream influence of the body for each value of the interaction parameter was characterized by the arbitrarily chosen distance between the point in the velocity profile at which $u=0.05$ and the body stagnation point. Figure 3 shows this length plotted as a function of the interaction parameter. The correlation with $N$ alone was not unexpected, for it is indicated by the

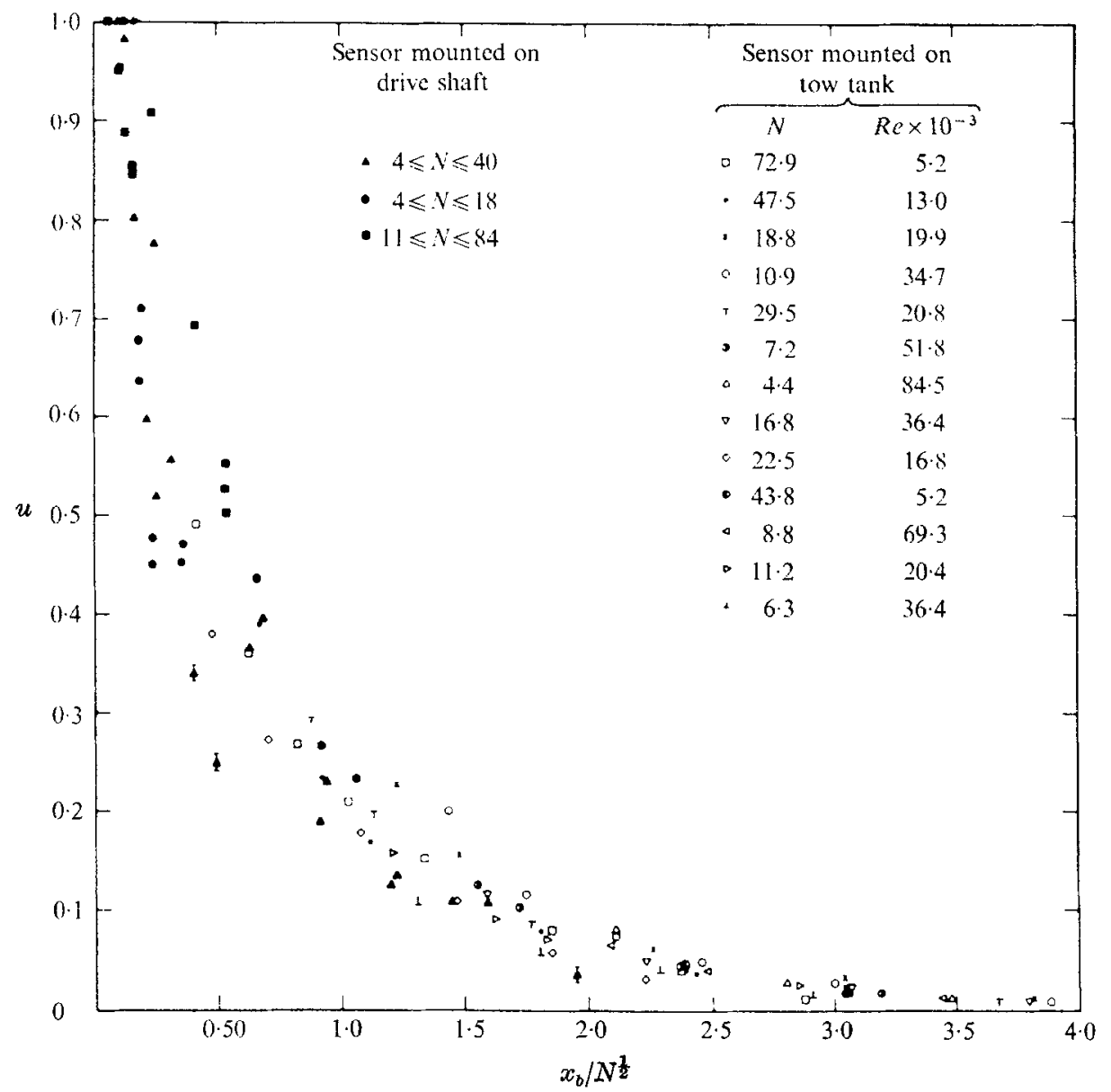

Figure 4. Normalized velocity on the flow centre-line $v s . x_{b} / N^{t}$.

equations of $\S 1$ and was found in the drag measurements of both Suzuki (1967) and Yonas (1966). Lines of slope $N$ and $N^{\frac{1}{2}}$ are plotted in figure 3, from which it can be seen that the disturbance length defined in this way is directly proportional to $N^{\frac{1}{2}}$.

The dependence of this length on $N^{\frac{1}{2}}$ suggests the use of $x_{b} / N^{\frac{1}{2}}$ as a new length scale. In figure 4, the results of velocity measurements made with the sensor mounted at the top of the tow tank and on the drive shaft are shown as functions of $x_{b} / N^{\frac{1}{2}}$. Although the data in the figure were obtained over ranges of more than an order of magnitude in both $N$ and $R e$, they form a single $u v s . x_{b} / N^{\frac{1}{2}}$ profile. The solid symbols represent the results of measurements made with the sensor 
mounted on the drive shaft, and lie within $0<x_{b} / N^{\frac{1}{2}}<1 \cdot 5$, which corresponds to more than $85 \%$ of the velocity change from 1 to 0 . All other symbols represent the results of measurements made with the sensor mounted on the tow tank. They lie in the range $0.5<x_{b} / N^{\frac{1}{2}}<4 \cdot 0$, which corresponds to $50 \%$ of the velocity change from 0 to 1 , so that a portion of the profile corresponding to approximately $35 \%$ of the total change in velocity is produced by the overlap of the two kinds of data.

In figure 5, the full profile is shown in a semi-logarithmic plot. Except for small

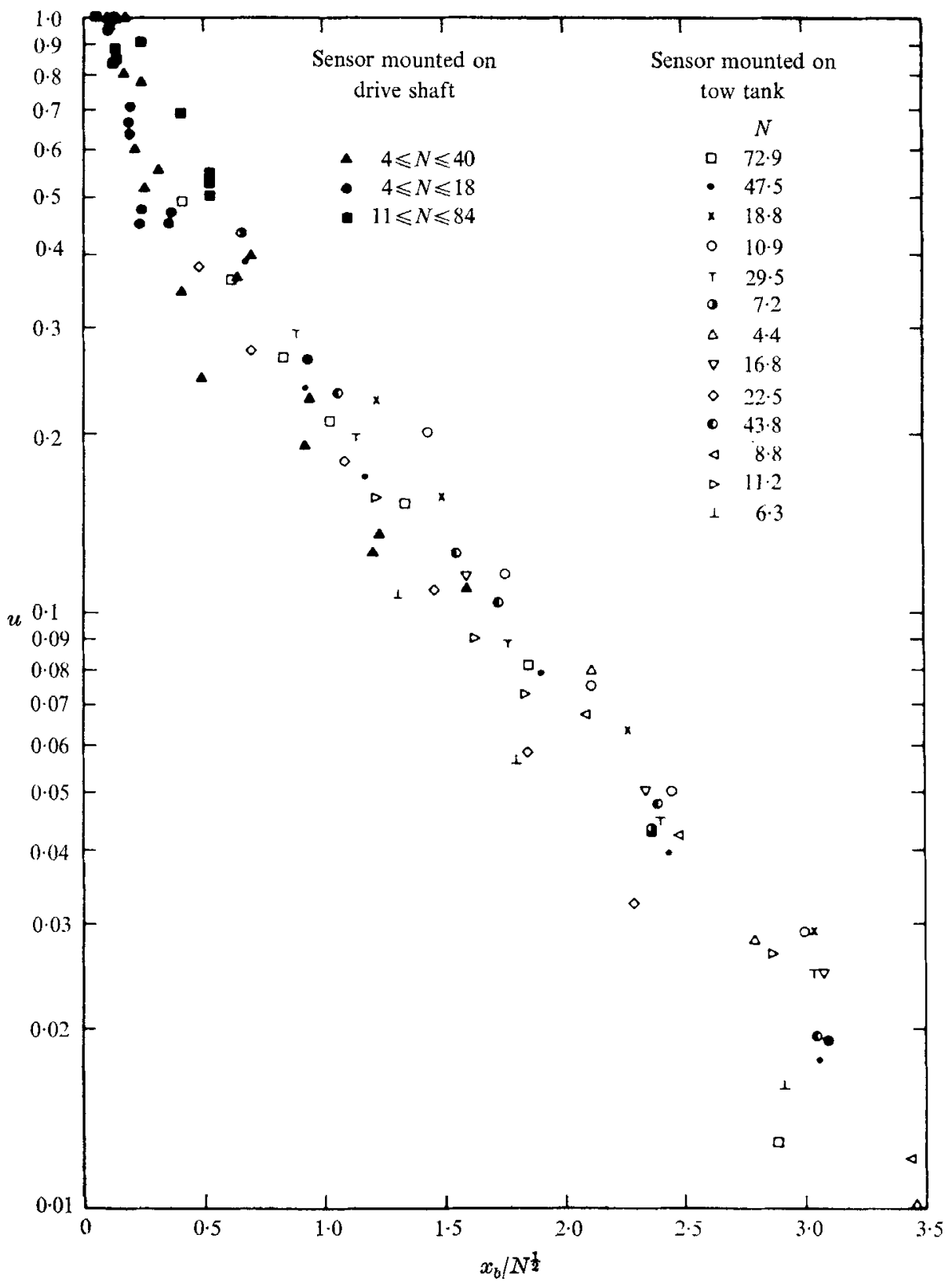

Figure 5. Normalized velocity on the flow centre-line $v s . x_{b} / N^{2}$. 
$x_{b} / N^{\frac{1}{2}}$, the data describe a straight line, which on such a plot indicates exponential behaviour. Away from the body, the axial velocity perturbations produced by it become exponentially small.

The results of the measurements made with the sensor tank-mounted at radial positions other than $r=0$, were first prepared in a form similar to that of figure 2 . For each value of $N$, a series of curves representing $u v s . x_{b}$ at each radial station was prepared. An example of such a plot is shown in figure 6. The interpretation of the measured quantity as the axial flow velocity is discussed below. A great

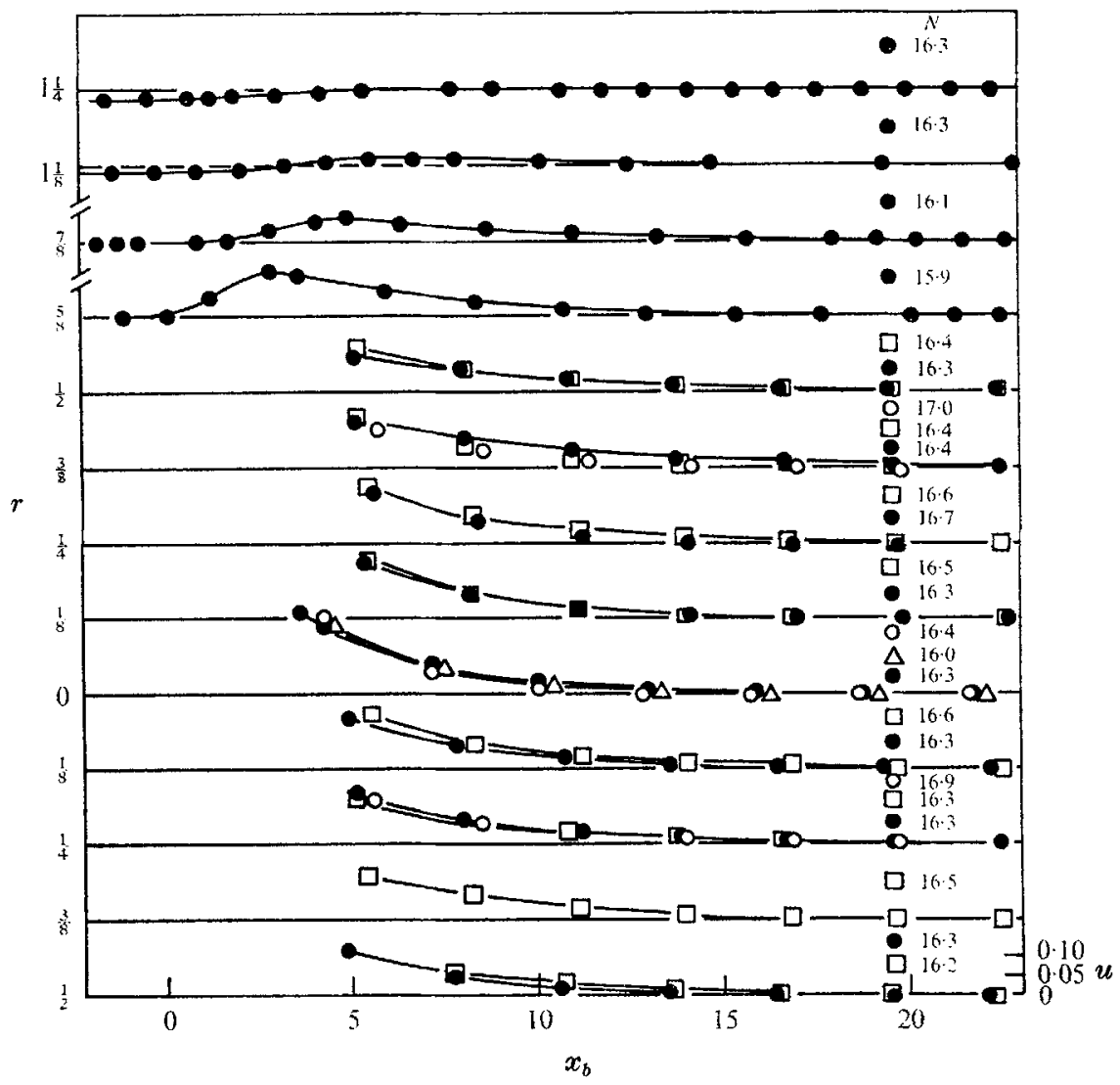

Figure 6. Examples of normalized velocity vs. distance from body measured at various radial positions in the flow.

deal of data of this kind was obtained over the full range of experimental conditions, and a series of plots such as that in figure 6 was obtained. By crossplotting these data it was possible to produce profiles of the axial velocity at various distances ahead of the Rankine body as shown in figure 7. Sets of profiles were obtained for values of $N$ ranging from 11 to 47 . These are shown in figure 8 . The bars on the curves in figure 8 indicate the scatter in the data used to define the curves.

The regions of the flow field in which velocities have been measured are: from $x_{b} \gg 1$ (far upstream) to within $x_{b}=0.5$ on $r=0$; from $x_{b} \gg 1$ to within $x_{b}=3$ for $0<r \leqslant 0.5$; and from $x_{b} \gg 1$ to $x_{b}<-2$ for $0.5<r<1.25$. 
The uncertainties associated with the directly measured quantities are used in Lake (1969) to provide estimates of the uncertainties in the final results of the experiments. The following is a brief review of those estimates.

Each value of the calibration parameter $X(P e) \propto[(\Delta T / q(0))-(\Delta T / q(P e))]$, was calculated using a pair of measurements made at different flow velocities, under the assumption that all other conditions were constant. The uncertainty in $X(P e)$

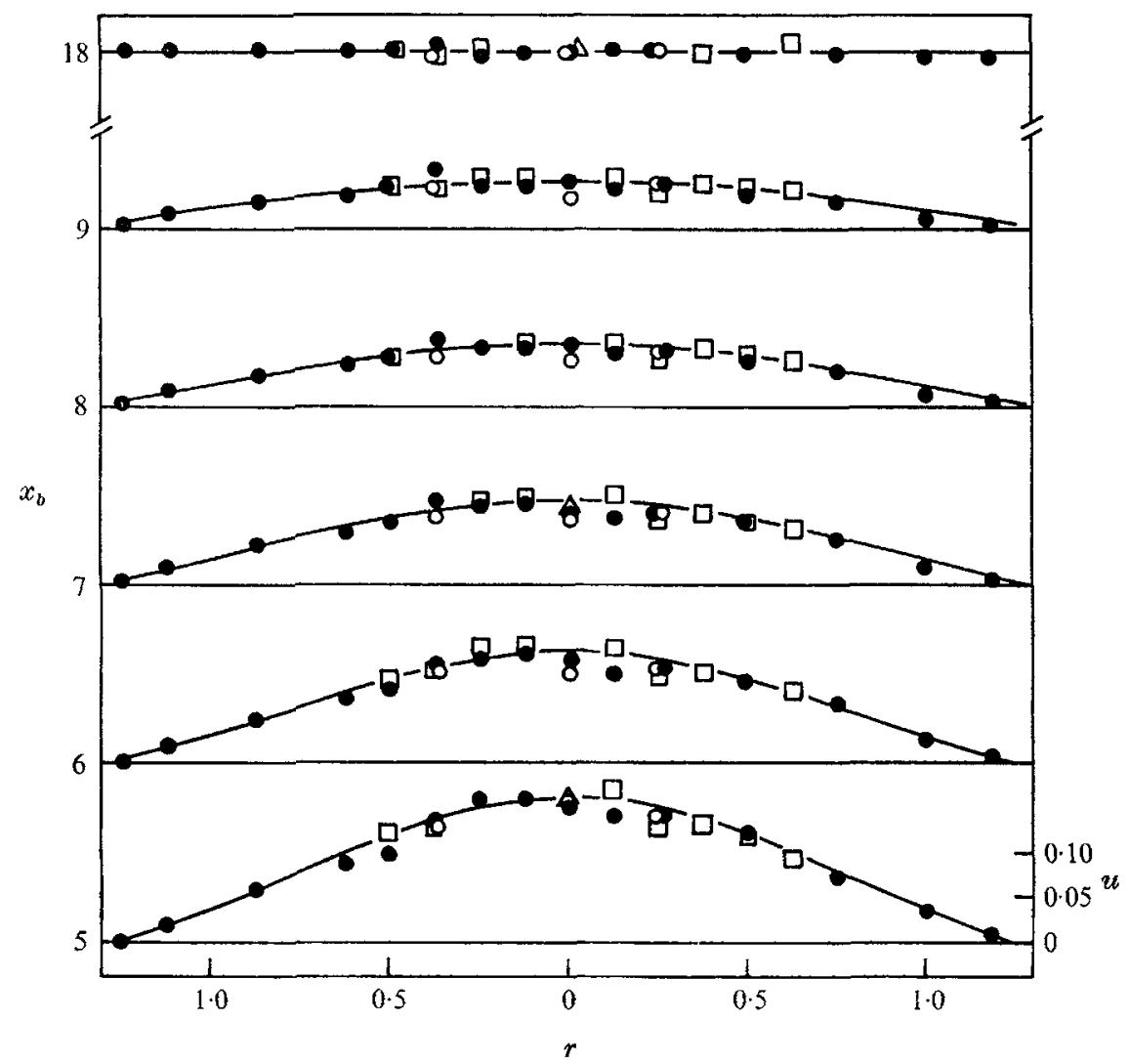

Figdre 7. Normalized axial velocity profiles for $N=16 \cdot 4$.

therefore depends not only upon the accuracy of the individual measurements, but also upon the amount by which the presumably constant conditions could have differed for a given pair of measurements. By considering the extent to which these conditions $\dagger$ could have changed, and the effects of such changes on $X(P e)$, the average uncertainty in $X(P e)$ is estimated to be $\pm 6 \%$. The scatter in the calibration curves, which is a measure of this same quantity, is of order $\pm 5 \%$.

Over most of the velocity range, when $X(P e)$ is used with the calibration curves to determine $P e$, the resulting average uncertainty in the latter is $\pm 7 \%$. At the highest velocities, where $X(P e)$ becomes an increasingly weak function of $P e$, this increases to the order of $\pm 15 \%$ to $\pm 20 \%$.

+ The important conditions are the mercury temperature and the sensor coating conditions. 
The uncertainty in the normalized velocity $u$ depends almost entirely on that associated with the $P e$ determined from the calibration curves. It varies according to the relative magnitudes of $u$ and $P e$, and the way in which $u$ is calculated. Most of the data were obtained using the sensor mounted on the tow tank. These estimates indicate that the values of $u$ calculated from such data are accurate to within $\pm 8 \%$, except for the smallest values $\left[u \leqslant O\left(10^{-2}\right)\right]$, which are known

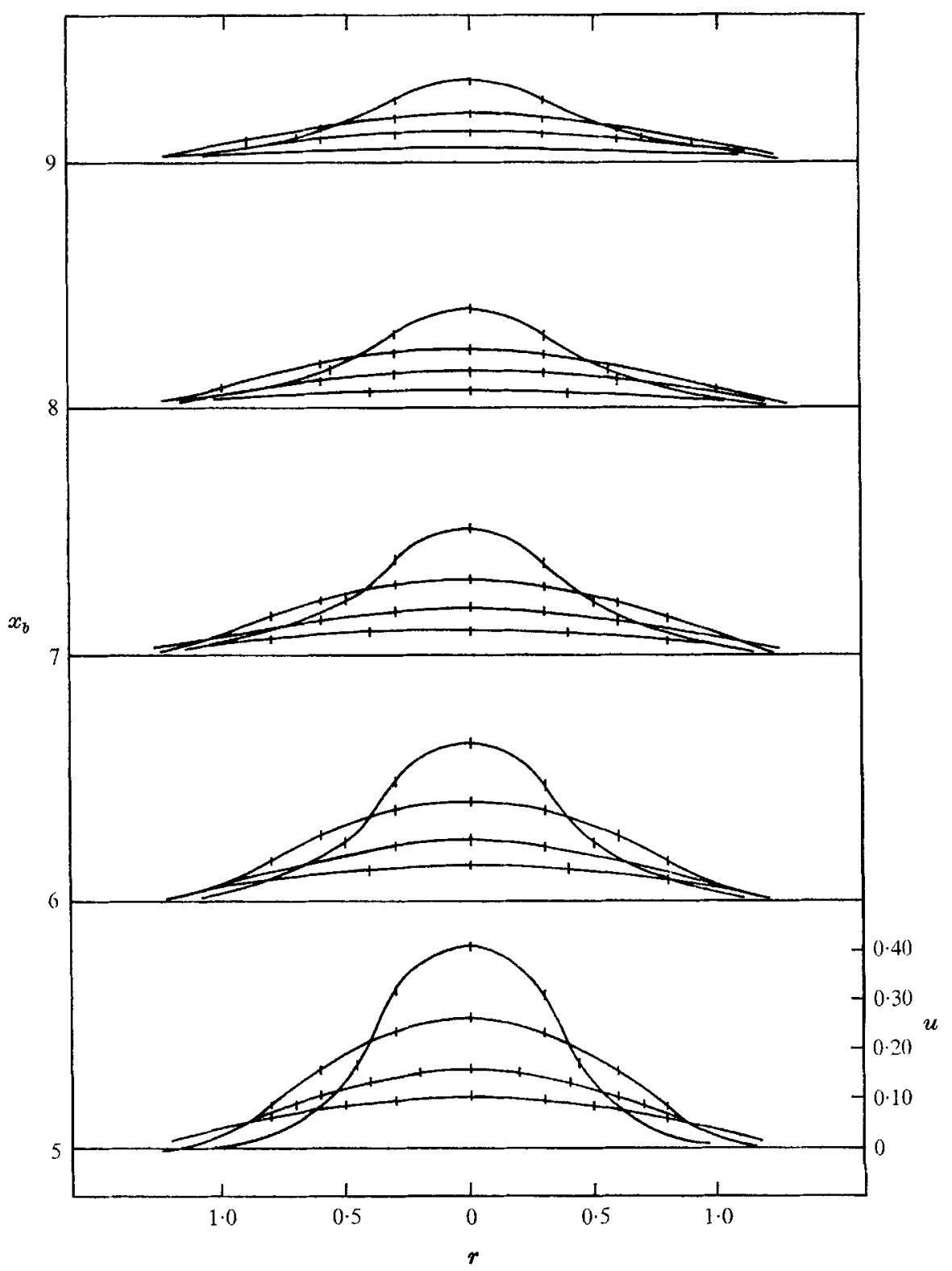

Figure 8. Normalized axial velocity profiles for $N=11 \cdot 2,16 \cdot 4,29$ and 47 . In each set of four profiles (at fixed $x_{b}$ values), the individual profiles are for $N=11 \cdot 2,16 \cdot 4,29$ and 47 , respectively, reading from bottom to top along $r=0$. 
only to within $\pm 10 \%$ to $\pm 40 \%$, depending on their magnitude. The estimated uncertainty of the normalized velocities calculated from the shaft-mounted sensor data varies from less than $\pm 7 \%$ to $\pm 15 \%$, except for a few values which are marked with error flags in figure 4 and are discussed in Lake (1969).

The sensor-to-body distances for the data taken with the sensor mounted on the tow tank could be calculated to within $\pm 4 \%$ for any given drive shaft speed. The relative position of the origin for different drive shaft speeds, however, could only be determined to within $\pm 0 \cdot 2$ body diameters. The effect of this is to increase the possibility of scatter in data obtained at equal values of $N$ using different drive shaft speeds.

When the sensor was used at radial positions other than that of the flow centreline, the signal was assumed to be related to only the axial component of velocity. As discussed in Lake (1969), this assumption was based on the fact that these sensors are insensitive to flow yaw angle in low Re mercury flows, and that in the experimental flow $v \ll u$. The assumption appears justified in view of the experimental results. They show that the data were not dependent on whether the sensor was perpendicular or parallel to the radial velocity, that the measured flow could satisfy the continuity equation, and that $v$, as inferred from the axial velocity data, satisfies $v \ll u$.

During the course of the experiments, operating procedures such as the sequence of sensor positions and controllable flow conditions were varied so as to ensure against the possibility of systematic errors in the results. In addition, it should also be noted that the profile defined by the data in figure 4 ( $\left.u v s . x_{b} / N^{\frac{1}{2}}\right)$ is produced by the overlapping of results which were obtained for widely different experimental conditions, and therefore depend in different ways upon the uncertainties in any one measurement. The fact that the agreement between them is quite good indicates that systematic errors are not present.

\subsection{Tow tank flow conditions}

The results of several measurements indicate that when the experimental flow was studied, it was in a steady state in the sense that it had become fully developed after its initiation. The results of the runs made with the sensor mounted on the drive shaft are one indication that this was true. They show that the velocity ahead of the body reached a constant value which was maintained throughout most of each run. In addition, experiments were performed, using a tank-mounted sensor, in which the starting distance (i.e. the distance between the model stagnation point and the sensor at the start of a run), was varied from 11 to 29 body diameters. The results which can be found in Lake (1969) show that for initial positions greater than 17, the data do not depend on starting position, whereas the starting position for all of the regular experiments was 29 body diameters. The measurements of magnetic field perturbations by Ahlstrom (1963) for $0 \cdot 6<N<4$, and the measurements of drag by Suzuki (1967) for $0<N<20$, made in the same facility, tend to confirm the conclusion that the experimental flow was fully established. Ahlstrom found that his results were independent of starting position for initial distances greater than 15, and Suzuki found that the drag force was constant during most of each run. 
The constraint imposed by the wall boundary conditions cannot have had a significant effect on the velocity field in the tow tank. Potential flow past a Rankine body in an unbounded fluid corresponds to a maximum radial velocity at $r=r_{\text {wall }}(=2 \cdot 75$ body diameters $)$ of only $0 \cdot 008$. The introduction of the magnetic force, which acts to suppress radial velocities, should reduce this further so that to a very good approximation at the values of $N$ used here, an unbounded flow would satisfy these experimental boundary conditions.

The other velocity boundary condition which must be considered is that imposed by the free surface at the top of the tow tank. Its influence must increase with increased $N$, since the length of the disturbance in front of the body increases with $N$. During all of the normal experiments which used a tank-mounted sensor, the sensor was located $7 \frac{1}{2}$ body diameters beneath the mercury surface. Additional experiments were performed in which all conditions were duplicated, including the position of the sensor with respect to the tow tank, except that the distance between the sensor and the mercury surface was varied by changing the mercury level in the tow tank. The results of these measurements are presented in Lake (1969) for distances of $2,4 \frac{1}{2}, 7 \frac{1}{2}$ and $8 \frac{1}{8}$ body diameters. For this range of surface positions they are independent of surface position for the whole range of $N$.

The length of the upstream disturbance (as defined in $\$ 3.1$ ) varies from about 5 body diameters at $N \sim 4.5$ to 15 body diameters at $N \sim 45$, increasing as $N^{\frac{1}{2}}$. The data taken in the normal way (with the surface $7 \frac{1}{2}$ body diameters above the sensor), produced velocity profiles which extend to within 4 to 5 body diameters of the stagnation point, so that all such data were obtained while the body was approximately 12 diameters or more away from the mercury surface. Only for values of $N$ greater than about 25 was the disturbance length greater than 12 diameters. Therefore, most of the 'normal' data were obtained while the body was at a distance from the surface greater than this disturbance length. On the other hand, nearly all of the data produced at the lowest surface levels were obtained while the upstream disturbance, so defined, was near enough to be interacting with the free surface. The results were the same for all these cases. If the flow ahead of the body had been significantly altered due to its interaction with the mercury surface, the effects should have become apparent when the normal results for the full range of $N$ were plotted together in the form $u v s . x_{b} / N^{\frac{1}{2}}$, as well as when the data for different surface levels were compared. In view of the actual results of these measurements, it must be concluded that during the experiments the body did not come near enough to the free surface for the latter to seriously affect the velocity field at the sensor position.

Finally, it should be pointed out that owing to the fluid displaced by the drive shaft, the flow in the tow tank is not strictly equivalent to free-stream flow past a stationary body. As a result, the normalized velocity $u$, referred to the displacement flow ahead of the body $(u=0)$, becomes negative downstream of the body $(u=-0.03)$ where the velocity relative to the tow tank is zero. 


\section{Discussion}

The experiments show that, compared to the zero field potential flow, the MHD flow for $N \geqslant O(1)$ has a large upstream disturbance extending far ahead of the body. The length of the disturbance, which grows as $N^{\frac{1}{2}}$, is 10 to 20 body diameters for the highest values of $N$ used here. $f$ On the other hand, the centreline velocity profiles show that only very much nearer the body is the fluid motion relative to the body actually reduced to the extent that it can be considered stagnated. Such flow did exist, however, just ahead of the body, as was dramatically demonstrated by the shaft-mounted sensor measurements. Although the sensor was sensitive to velocities as small as a few per cent of the drive shaft velocity, it produced a steady zero-velocity signal when mounted $0.5 \mathrm{in}$. ahead of the body at $N \gtrsim 17$, and when mounted 1 in. ahead of the body at $N \gtrsim 35$. The measurements do not provide sufficient data to describe the dependence of the length and shape of this stagnated portion of the flow on $N$. However, they do show that the flow ahead of the body consists of a relatively short region of stagnant fluid preceded by a much longer region in which the fluid velocity rapidly approaches that of the free-stream flow. Only if the former grows as $N^{\frac{1}{2}}$, will the correlation of $u$ and $x_{b} / N^{\frac{1}{2}}$ in figure 4 remain valid for all $N$. The stagnated region, however, could be increasing in length at a faster rate such as directly with $N$. In such a case, for increased $N$ the transition from zero to free-stream velocity would have to take place within a region no longer similar to the one measured here, or within one in which the distance $x_{b}$ is referred to the front of the stagnant region and not to the body. Such departures of the flow pattern from that measured in these experiments could only occur for values of $N$ much greater than those obtained here. They would imply that the limiting flow for $N \rightarrow \infty$ is approached only very slowly-even as $N$ is increased by orders of magnitude.

The full axial velocity results show that, with increased $N$, the magnitude of the velocity defect at a fixed point ahead of the body increases on and near the flow centre-line. It increases more slowly, or may even decrease, at larger radial positions. In other words, at the same time that the total velocity defect increases, it also becomes more concentrated about the axis of symmetry. If the radial position at which the defect is one-half its maximum is used as a measure of the width of each velocity defect profile, the width of the disturbance slowly decreases at a fixed axial position as $N$ is increased. For a fixed $N$, it grows very slowly with increased distance from the body.

An indication of the current distribution can be obtained using the fact that the current density at a point in the flow is just proportional to the radial velocity at that point. The average radial velocity between $x_{b}$ and $x_{b}+1$ at $r$ was calculated by graphically integrating the continuity equation

$$
v=-\mathbf{1} / r \int_{0}^{r}(\partial u / \partial x) r \partial r
$$

using the profiles of axial velocity to provide $\partial u / \partial x$. The results, shown in figure 9 , provide an estimate of the magnitude and distribution of $v$ and $j$. These quantities

$\dagger$ The corresponding length for flow at $N=0$ is 0.85 body diameters. 
increase from zero at $r=0$ almost linearly with $r$, reflecting the weak $r$-dependence of $\partial u / \partial x$ for small $r$. As $r \rightarrow O(1)$, the current density and radial velocity reach maxima and then decrease with further increases in $r$. Typical radial velocities in the flow are an order of magnitude smaller than the characteristic velocity defect at the same axial position.

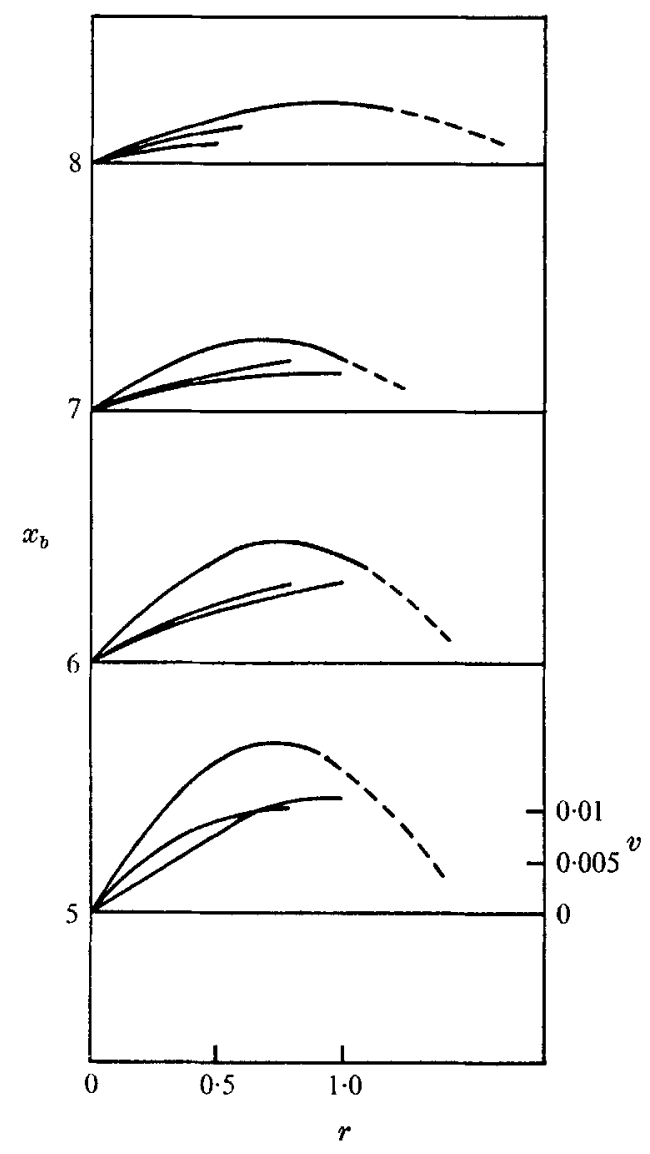

Fiqure 9. Normalized radial veloeity profiles for $N=11.2,16.4$ and 29 . In each set of three profiles (at fixed $x_{b}$ values), the individual profiles are for $N=11 \cdot 2,16.4$ and 29 , respectively, reading from bottom to top near $r=0$.

These experimental results provide the magnitude and distribution of $u, v$ and $j$ in the flow ahead of the body for $N>10$. The current layer model referred to in $\S 1$ describes these same quantities in the same flow region under the assumption that $N \gg 1$. In the model flow, there exist well-defined current layers, centred about $r=\frac{1}{2}$, which maintain the radial pressure gradient necessary to separate the outer free-stream flow from a slug of nearly stagnated fluid ahead of the body. The merging of the layers, which terminates the stagnant region, takes place a distance $x=O(N)$ ahead of the body. In the limit $N \rightarrow \infty$, for $N / R e \rightarrow 0$, the model flow becomes undisturbed free-stream flow past an infinitely long slug of fluid bounded by infinitesimally thin current sheets. Therefore, while 
the measured flow does not preclude the possibility of such a limiting form, it does not fit the current layer model. Instead of well-defined current layers, there are broad regions of maximum current density. The length of the upstream disturbance grows as $N^{\frac{1}{2}}$. The small portion of it which contains stagnated flow grows with $N$ at an undetermined rate, but is definitely not of $O(N)$ in length. Although the entire disturbance becomes more 'slug-like' in that it becomes more concentrated about $r=0$ as $N$ increases, it is not a slug of very slowly moving fluid. The current layer model describes a region containing a slug of nearly stagnant fluid bounded by current layers, but provides no description of the intermediate region which must exist between the slug and the upstream fluid. In the measured flow it is found that the stagnant region, which could not be measured in detail, is short, and that most of the disturbance consists of a region of transition from zero to free-stream velocity.

The experimental flow can be described further by referring to the generalized Bernoulli law for inviscid MHD flows derived by Tamada (1962),

$$
\begin{aligned}
\mathbf{q} \cdot \nabla \boldsymbol{H} & =\mathbf{q} \cdot \nabla\left[\frac{1}{2}\left(u^{2}+v^{2}\right)+p\right] \\
& =N \mathbf{q} \cdot(\mathbf{j} \times \mathbf{B})=-N j^{2} .
\end{aligned}
$$

The Bernoulli function $H=\frac{1}{2}\left(u^{2}+v^{2}\right)+p$, is constant along streamlines on which $j=0$, and must decrease along all streamlines on which $j \neq 0$. This means that since $v \sim j$, closed streamlines are not possible in steady flow, and also that the maximum pressure in the flow is normal stagnation pressure $\left(P_{0}=\frac{1}{2}\right.$ in this notation) at the stagnation point. In both the non-magnetic and MHD flows, the static pressure along $r=0$ rises from its free-stream value (zero) to this stagnation pressure at the body. In both cases the axial pressure gradient is balanced only by the axial fluid deceleration. The difference between the two flows appears in the radial equation of motion. Radial pressure gradients in the ordinary flow can only be supported by the inertia terms, but in the MHD flow for $N \gg 1$ these may be neglected, so that the equation becomes $\partial p / \partial r \simeq-N v$. The ability of the radial flow to support a radial pressure gradient is enhanced, while the net radial flux of fluid remains unchanged (for a given free-stream velocity), and the maximum possible radial pressure drop from $r=0$ to $r \gg 1$ is constant. Qualitatively, the magnetic force which acts on the fluid when it crosses field lines tends to decrease the radial flow near the body and increase it away from the body. Thus, it straightens the streamlines and increases the axial distance over which the pressure rises from $p=0$ upstream to $p \leqslant \frac{1}{2}$ at the body.

Along the axis of the flow $v=j=0$, and the Bernoulli function is constant, $H=\frac{1}{2}$, so that the local pressure is directly related to the known axial velocity, $p(x, 0)=\frac{1}{2}-\frac{1}{2} \hat{C}^{2}(x, 0) \dagger$. Therefore, the pressure change along $r=0$ occurs over an axial distance proportional to $N^{\frac{1}{2}}$. An example of this pressure profile for $N=29$ is shown in figure 10, along with the corresponding profile for potential flow.

$\uparrow$ The results of the experiments are presented in terms of the normalized velocity defect in the free-stream flow, so that the velocity referred to above is $\widetilde{U}=1-u_{\text {defect }}$ and is the normalized fluid velocity relative to the body. 
The pressure difference which exists between $r=0$ and the flow at large $r$ is maintained by the net magnetic force,

$$
-N \int_{0}^{r} v(x, r) \partial r
$$

at $x$. Lacking an analytical expression for the distribution of radial velocity, the area under the velocity profiles of figure 9 can be used to provide an estimate of the magnitude of this force. For example, at $N=29$, the values estimated in this way are 0.36 at $x_{b}=5,0.26$ at $x_{b}=6$ and 0.145 at $x_{b}=8$. The corresponding changes in pressure from figure 10, assuming $p=0$ in the outer flow, are approximately $0 \cdot 24,0 \cdot 18$ and $0 \cdot 13$. $†$ These admittedly crude estimates are cited only to demonstrate that the radial velocities which correspond to the measured axial velocity profiles are of the correct order, and sufficient, to support the radial pressure gradients which must exist in the flow.

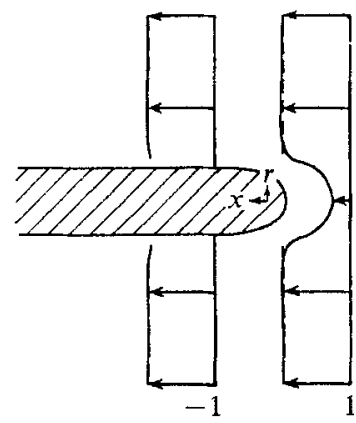

Mean velocity $=1 \cdot 03$
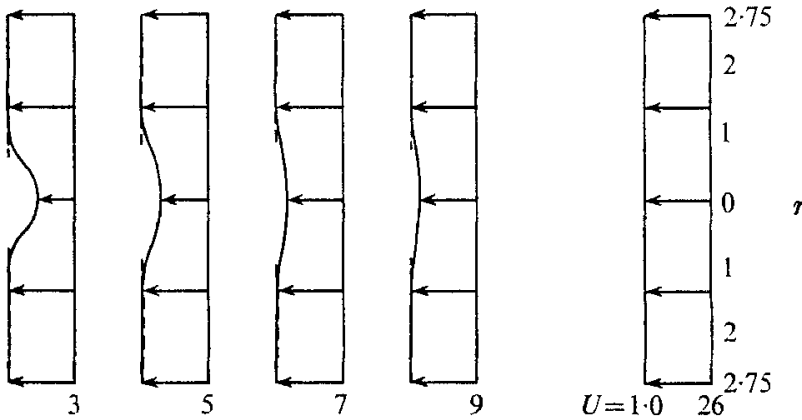

Axial velocity profiles body-fixed co-ordinate system

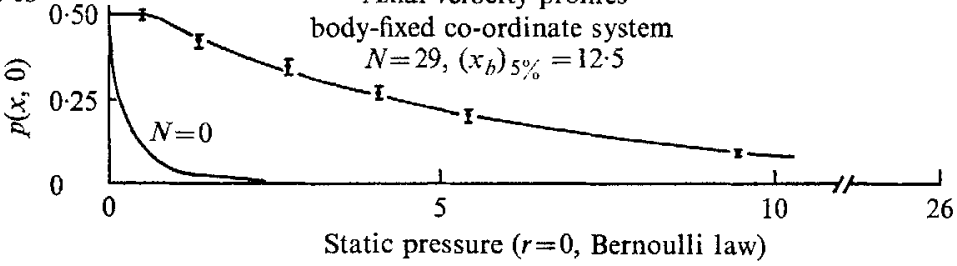

$x_{b}$

Figure 10. Flow in the tow tank for $N=29$.

It may be noted here that had the radial velocities been confined to relatively thin layers, their maximum values (which were $0.017,0.012$ and 0.006 at the positions referred to above for $N=29$ ) would have had to have been considerably higher at the same axial positions and same $N$. And, finally, that if layers of some kind are assumed to exist very near the body where there is a region of stagnated fluid, they must be able to support a radial pressure change of order $0 \cdot 5$. Given an estimate of the thickness of the layers in this region, an approximate mean velocity through them would then be known. However, despite the fact that near the body the distribution of radial velocity must become reasonably concentrated around $r=\frac{1}{2}$, the flow in this region (where the stagnated flow,

$\dagger$ These values should be slightly low because in the outer flow $p \rightarrow-0.03$ downstream of the body owing to the blockage effect of the body in the tow tank. 
the curved body face, and the outer flow come together) is certainly too complex to be described in terms of a simple current layer.

The effects of the Joule dissipation on the pressure and velocity in the flow can be considered by referring again to the Bernoulli law. The stagnation pressure, $P_{0}=p+\frac{1}{2}\left(u^{2}+v^{2}\right)$, is decreased on all streamlines which have passed through regions of non-zero current density. The decrease at any point in the flow is equal to the total Joule dissipation which has occurred upstream of the point along the streamline passing through it,

$$
\Delta P_{0}=-N \int j^{2} d s=-N \int v^{2} d s
$$

Since the stagnation pressure can only decrease, and the amount of Joule dissipation which occurs along different streamlines varies, radial as well as axial stagnation pressure gradients occur in the flow. At axial positions ahead of the body the stagnation pressure falls from $\frac{1}{2}$ on $r=0$ to lower values at $r>0$ in the region where streamlines which have undergone dissipation pass, and then rises again to $\frac{1}{2}$ at large $r$ where there are streamlines on which the radial velocity has been continuously zero. As shown by Tamada (1962), far downstream of the body where the flow again becomes uniform in the sense that $v=0$, there can be no radial static pressure gradient, the axial velocity must be directly related to the stagnation pressure, and there is a vortical wake due to the loss in stagnation pressure suffered by the flow along streamlines which have experienced Joule dissipation. For Re large, but not infinite, this wake will ultimately diffuse to produce uniform flow far downstream.

Because of the stagnation pressure variations in the flow, the velocity measurements cannot be used directly to determine static pressures. This is particularly true over the front of the body near $r=\frac{1}{2}$ where the stagnation pressure must be close to its minimum value. Measurements of static and stagnation pressures over the surface of a sphere and a sphere-and-afterbody combination in alignedfields MHD flow, for $1.5<N<40$, have been made by Maxworthy $(1968,1969)$. These show that the stagnation pressure in the flow past the sphere, at the position corresponding to $r=\frac{1}{2}$ on the half-body used here, is from 40 to $90 \%$ less than its free-stream value and decreases as $N^{\frac{1}{2}}$. At the same position, static pressures are found to be of $O(1)$ and negative, so that the corresponding velocities must be greater than free stream by as much as $50 \%$. The resultant net pressure force on the front of the sphere rises only to about $C_{D}=0.5$, at $N \sim 12$ for the sphere and at $N \sim 5$ for the combination body, and then falls toward zero as $N$ is increased further, owing to the effect of the increasingly large contribution of the negative pressure. In addition, largenegative pressures near the body can only exist if maintained by a magnetic force, so that the flow past the body must contain negative radial velocities. These results, which were obtained for finite bodies rather than a simple half-body shape as used here, are cited to demonstrate the possible effects of the stagnation pressure losses due to Joule dissipation in such flows, and to introduce a discussion of whether such an effect could occur in the flow under consideration here.

Suzuki (1967) has measured the drag of a Rankine half-body in these flows. 
His measurements show that, as $N$ is increased from $N \ll 1$, the drag increases linearly with $N$ to a value of $C_{D} \sim 0.5$ at $N=6$. For $N>6, C_{D}$ increases more slowly with increased $N$, and appears to be approaching $C_{D} \sim 0.8$ asymptotically for $N>20$. Although at low $N$ there are certainly regions of negative pressure on the front of the body (as there are for $N=0$ ), these measurements indicate that they are probably not increasing in strength with increased $N$. The highest drag possible for a half-body in these flows is $C_{D} \rightarrow 1.0$ as $N \rightarrow \infty$, for which the entire frontal area must be at free-stream stagnation pressure. The velocity measurements indicate that it is unlikely that this condition exists in the flow even at $N>20$, so $C_{D} \sim 0.8$ can occur only if there is very little negative pressure on the body. Another indication of the pressure near the body comes from some velocity measurements made in the flow downstream of the body. Owing to physical limitations imposed by the experimental apparatus, these could only be made for $0.625 \leqslant r \leqslant 1.25$ and for only 2 to 3 body diameters past the stagnation point. Nevertheless, the results are sufficient to indicate that axial velocities in this region were not greater than free stream, but were, in fact, smaller. Typical results of such measurements are included in figures 6 and 10. On the basis of these drag and velocity measurements, it must be concluded that the static pressure on the body near $r=\frac{1}{2}$ is not large and negative, but is probably close to zero. The stagnation pressure in this region would then be of order

$$
P_{0}=\frac{1}{2} u^{2}+p<\frac{1}{2},
$$

where $u<1$ and $p$ is approximately zero or at most slightly negative. It should be noted here that because the upstream flow is being forced away from the axis in order to pass the body, the flow outside the disturbance must be accelerating. Since the current regions are broad and extend well beyond $r=\frac{1}{2}$, especially at large $x_{b}$, flow at fairly large radial distances is turned and accelerated. As a result, the regions of accelerated flow and stagnation pressure loss past the body are widespread. There is no large radial gradient in axial velocity or, except possibly very close to the body, in stagnation pressure.

\section{Conclusion}

Velocities in aligned-fields MHD flow ahead of a semi-infinite Rankine body have been measured over a wide range of $N$. Centre-line flow velocities have been measured to within one-half body diameter of the stagnation point, and velocity profiles across the flow to within about five body diameters. It was found that with increased $N$, the upstream disturbance tends to become more confined radially within the region directly ahead of the body, and that its length increases as $N^{\frac{1}{2}}$. The flow was found to contain a region of stagnant fluid ahead of the body, and a much longer region over which the transition is made from free-stream conditions to conditions near the body. The rate at which the length of the stagnant region increases with $N$ was not determined. However, the region was found to be much shorter in length than is predicted by a thin current layer model. The radial gradients of velocity components, pressures and current density were found to be considerably smaller than suggested by such a model. 
The results are consistent with a drag coefficient which increases as $N$ is increased and approaches $O(1)$. In the corresponding flow past the body, the radial velocity and current density go to zero, and there are small radial gradients in the axial velocity and the stagnation pressure.

Although a reasonably complete description of the flow has been obtained using the measurements and the inviseid equations of motion for $N \gg 1$ and $R m \ll 1$, a different flow may evolve as $N \rightarrow \infty$. The limiting flow which develops, as $N$ is greatly increased over the values used here, will depend on how the length of the stagnant region increases in relation to the transition region ahead of it. The interaction parameter may have to be much larger than in these experiments before such a limit is approached.

This work was supported by the Office of Naval Research under contract no. N00014-67-A-0094-001 and submitted in partial fulfilment of the requirements for the degree of Doctor of Philosophy in Aeronautics, California Institute of Technology. The guidance of Professor H.W. Liepmann throughout the course of the work is gratefully acknowledged. The author would also like to thank Professor B.H. Suzuki of the University of Southern California and Professor J.A. Shercliff of the University of Warwick for many helpful discussions.

\section{REFERENCES}

Aнrstrom, H. G. 1963 Experiments on the upstream wake in magneto-fluid dynamics. J. Fluid Mech. 15, 205.

ChILdress, S. $1963 a$ On the flow of a conducting fluid of small viscosity. JPL Tech. Report, no. 32-351.

Childress, S. $1963 b$ The effect of a strong magnetic field on two-dimensional flows of a conducting fluid. J. Fluid Mech. 15, 429.

LAKE, B. M. 1969 Velocity measurements ahead of a semi-infinite body in magnetohydrodynamic flow with aligned fields. Ph.D. Thesis, California Institute of Technology.

Liepmann, H. W., Hoult, D. P. \& Ahlstrom, H. G. 1962 Concept, construction, and preliminary use of a facility for experimental studies in magneto-fluid dynamics. Mizellen der Angewandten Mechanick, p. 175. Berlin: Akademie.

MAxcoLM, D. G. 1969 Some aspects of turbulence measurements in liquid mereury using cylindrical quartz-insulated hot-film sensors. J. Fluid Mech. 37, 701.

MaxwoRthy, T. 1962 Measurements of drag and wake structure in magneto-fluid dyanamic flow about a sphere. Proc. Heat Transfer and Fluid Mech. Inst. p. 197. Stanford University Press.

Maxworthy, T. 1968 Experimental studies in magneto-fluid dynamics: pressure distribution measurements around a sphere. J. Fluid Mech. 31, 801.

Maxworthy, T. 1969 Experimental studies in magneto-fluid dynamies: flow over a sphere with a cylindrical afterbody. $J$. Fluid Mech. 35, 411.

SAJBEN, M. 1965 Hot-wire anemometer in liquid mercury. Rev. Sci. Instrum. 36, 945.

Shercliff, J. A. 1965 A Textbook of Magnetohydrodynamics. Pergamon.

Suzuki, B. H. 1967 Magneto-fluid dynamic drag measurements on semi-infinite bodies in aligned fields. Ph.D. Thesis, California Institute of Teohnology.

TAMADA, K. 1962 Flow of a slightly conducting fluid past a circular cylinder with strong, aligned field. Phys. Fluids, 5, 817.

Yonas, G. 1966 Aligned-fields, magneto-fluid dynamic flow past bodies. Ph.D. Thesis, California Institute of Technology. 